Chemical Physics 142 (1990) 431-443

North-Holland

\title{
ACCUMULATION OF DODCI P ISOMERS BY LASER EXCITATION
}

\author{
W. BÄUMLER and A. PENZKOFER \\ Naturwissenschaftliche Pakultät II - Physik, U'niversität Regensburg, D-8400 Regensburg, FRG
}

Received 10 October 1989

\begin{abstract}
The accumulation of molecules in the P-isomer ground state of DODCI in ethylene glycol is studied experimentally and theoretically. The $\mathrm{P}$-isomer $\mathrm{S}_{0}$ state is populated by $\mathrm{S}_{0}-\mathrm{S}_{1}$ absorption of $\mathrm{cw}$ argon ion laser light or dye laser light at various wavelengths. The population is probed by the absorption of a weak He-Ne laser. The recovery to the thermal ground-state equilibrium of $\mathrm{N}$ and $\mathrm{P}$ isomers is determined by measuring the temporal probe light absorption after pump laser switch-off. A steady-state level population theory is derived and applied to determine P-isomer $S_{0}-S_{1}$ absorption cross sections and transfer efficiencies between $\mathbf{N}$ isomers and $\mathbf{P}$ isomers.
\end{abstract}

\section{Introduction}

The dye DODCI (3,3'-diethyloxadicarbocyanine iodide) is widely used as saturable absorber in picosecond and femtosecond rhodamine $6 \mathrm{G}$ dye lasers [1-9]. The excitation of DODCI to the $S_{1}$ state leads to photoisomerization and to the accumulation of molecules in the $P$-isomer $S_{0}$ electronic ground state [10-13]. The groundstate $\mathrm{P}$-isomer to $\mathrm{N}$-isomer recovery time $\tau_{\mathrm{PN}}^{0}$ is of the order of a few milliseconds at room temperature $[11,12,14]$. In some papers $[2,8,15,16]$ the bleaching of the ground-state P-isomer absorption is thought to be responsible for the picosecond and femtosecond pulse generation in passively mode-locked rhodamine $6 \mathrm{G}$ dye lasers. For an evaluation of the P-isomer contributions to the mode locking it is necessary to know the accumulation dynamics of DODCI in the P-isomer ground state by photoisomerization. Accumulation studies in the DODCI absorber jet of a colliding pulse mode-locked rhodamine $6 \mathrm{G}$ dye laser are reported in ref. [13].

In this paper the accumulation of population in the $\mathrm{P}$-isomer $\mathrm{S}_{0}$-ground state of DODCI in ethylene glycol is studied. At various wavelengths in the region between $\lambda_{L}=488$ and $620 \mathrm{~nm}$. the $S_{1}$ state of the dye is excited by sither a cw argon ion laser. a cw rhodamine 110 dye laser, or a cw rhodamine $6 \mathrm{G}$ dye laser. The $\mathrm{P}$-isomer $\mathrm{S}_{0}$ state population is probed by transmission measurement of a He-Ne laser $\left(\lambda_{\mathrm{pr}}=632.8 \mathrm{~nm}\right)$. The probe laser wavelength is at the long-wavelength side of the $P$-isomer $S_{0}-S_{1}$ absorption peak $\left(\lambda_{\max }^{p} \approx 620 \mathrm{~nm}\right)$ and far off the $\mathrm{N}$-isomer $\mathrm{S}_{0}-\mathrm{S}_{1}$ absorption peak $\left(\lambda_{\max }^{N} \approx 585 \mathrm{~nm}\right)[17,18]$. From the measured probe laser transmissions the mole fractions of $P$ isomers in the $S_{0}$ ground state, $x_{P}^{0}$, are calculated. Population accumulation in triplet states was checked by probe light transmission measurements at $667 \mathrm{~nm}$ and found to be negligible.

In a separate experiment the ground-state $\mathrm{P}$-isomer to $\mathrm{N}$-isomer back relaxation rate, $k_{\mathrm{PN}}^{0}=\left(\tau_{\mathrm{PN}}^{0}\right)^{-1}$, is determined by probe pulse transmission recovery measurement after pump pulse switch off.

The experimental results are compared with a steady-state level population theory in order to obtain information on unknown $\mathrm{P}$-isomer $\mathrm{S}_{0}-\mathrm{S}_{1}$ absorption cross sections and on unknown $\mathrm{N}$-isomer to $\mathrm{P}$-isomer and $\mathrm{P}$ isomer to $\mathrm{N}$-isomer transfer rates. 


\section{Fundamentals}

The photoisomerization dynamics of DODCI is described by the $S_{0}$ and $S_{1}$ potential energy surface diagram of fig. 1 [18]. The $S_{0}$-state activation energy $E_{A}^{0}$ was determined in ref. [11] (DODCI in ethanol). The level position $E_{\mathrm{P}}^{0}$ of the $\mathrm{P}$ isomer in the $\mathrm{S}_{0}$ ground state was measured in refs. [17,18]. The $\mathrm{S}_{1}$-state activation energies, $E_{\mathrm{A} . \mathrm{N}}^{1}$ and $E_{\mathrm{A} . \mathrm{P}}^{1}$, were reported in ref. [18]. The barrier height, $E_{\mathrm{A} . \mathrm{I}}^{1}$, of the intermediate $\mathrm{S}_{1}$-state level is still unknown. The results of ref. [18] are compatible with $0 \leqslant E_{\mathrm{A}, 1}^{1} \leqslant E_{\mathrm{A} . \mathrm{N}}^{1}$. In the case of $E_{\mathrm{A}, 1}^{1}=0$ the $\mathrm{S}_{1}$-state threevalley model reduces to a double-valley model.

A pump laser of frequency $\nu_{L}$ excites molecules from the $S_{0}$ ground state of the $N$ and $P$ isomers to the corresponding $S_{1}$ states. A fast vibrational relaxation occurs in the $S_{1}$ state [19]. The molecules may transfer partially between the $\mathrm{N}$ - and $\mathrm{P}$-isomer conformations in the $\mathrm{S}_{1}$ state (rates $k_{\mathrm{NP}}^{1}$ and $k_{\mathrm{PN}}^{1}$ ). The $\mathrm{S}_{1}$-state $\mathrm{N}$-isomer radiative relaxation rate to the $\mathrm{N}$-isomer $\mathrm{S}_{0}$ ground state is $k_{\mathrm{rad}, \mathrm{N}}$, and the nonradiative $\mathrm{N}$-isomer $\mathrm{S}_{1}$-state to $\mathrm{S}_{0}$-state relaxation rate is $k_{\mathrm{nr} . \mathrm{N}}$. The $\mathrm{S}_{1}$-state $\mathrm{P}$-isomer relaxation rates to the $\mathrm{P}$-isomer $\mathrm{S}_{0}$ ground state are $k_{\mathrm{rad}, \mathrm{P}}$ and $k_{\mathrm{nr}, \mathrm{P} \text {. The }}$ total intra-isomer $S_{1}-S_{0}$ decay rates are

$k_{\mathrm{N}}=k_{\mathrm{rad}, \mathrm{N}}+k_{\mathrm{nr}, \mathrm{N}}=\tau_{\mathrm{N}}^{-1}=\left(\tau_{\mathrm{rad}, \mathrm{N}} q_{\mathrm{N}}\right)^{-1}$,

$k_{\mathrm{P}}=k_{\text {rad.P }}+k_{\text {nr.P }}=\tau_{\mathrm{P}}^{-1}=\left(\tau_{\text {rad.P }} q_{\mathrm{P}}\right)^{-1}$,

where $\tau_{N}\left(\tau_{P}\right)$ is the fluorescence lifetime of the $N$ isomer $(P$ isomer $) . \tau_{\text {rad.N }}\left(\tau_{\text {rad.P }}\right)$ is the radiative $S_{1}$-state lifetime of the $\mathrm{N}$ isomer ( $\mathrm{P}$ isomer). $q_{\mathrm{N}}$ and $q_{\mathrm{P}}$ are the fluorescence quantum efficiencies of the $\mathrm{N}$ and $\mathrm{P}$ isomers, respectively. These quantities have been determined previously by temperature-dependent fluorescence quantum distribution measurements [18] and they are listed in table 1.

The molecules may transfer partially between the $\mathrm{N}$ - and $\mathrm{P}$-isomer conformations in the $\mathrm{S}_{1}$ state with the rates $k_{\mathrm{NP}}^{1}$ and $k_{\mathrm{PN}}^{\prime}$. An $\mathrm{S}_{1}$-state to $\mathrm{S}_{0}$-state inter-isomer $\mathrm{N}$-isomer to $\mathrm{P}$-isomer and $\mathrm{P}$-isomer to $\mathrm{N}$-isomer relaxation may occur with the rates $k_{\mathrm{NP}}^{10}$ and $k_{\mathrm{PN}}^{10}$, respectively.

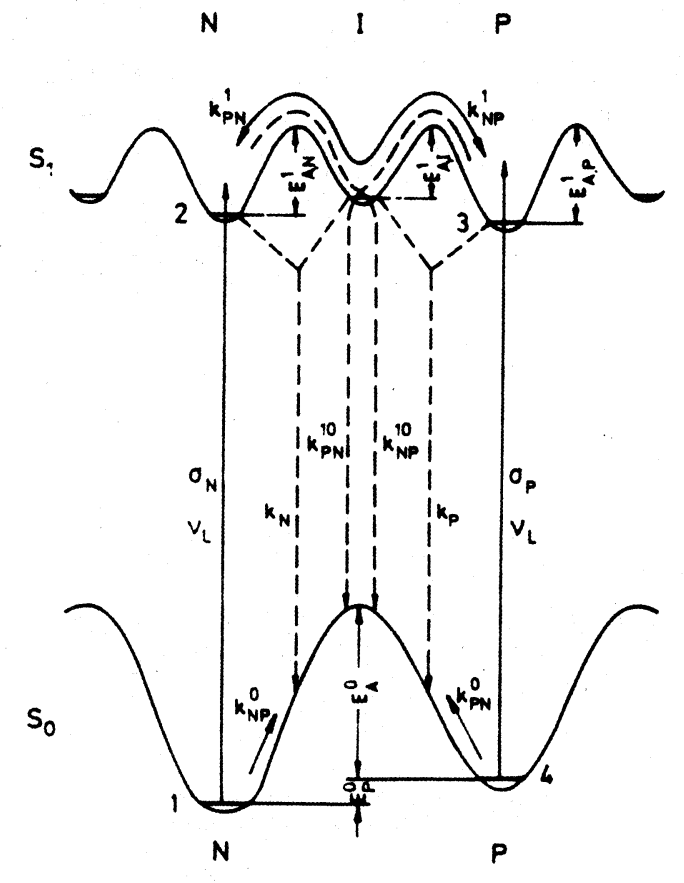

Fig. 1. Potential energy curve diagram of DODCI. Parameters are explained in the text. 
Table 1

Parameters of DODCI in ethylene glycol at room temperature $\left(\theta=25^{\circ} \mathrm{C}\right)$. Dye concentration $C=2.5 \times 10^{-5} \mathrm{~mol} / \mathrm{dm}^{3}$

\begin{tabular}{llll}
\hline & Parameter & Value & Ref. \\
\hline$\tau_{\text {rad.. }}(\mathrm{ns})$ & 2.35 & {$[18]$} \\
$q_{\mathrm{N}}$ & 0.55 & {$[18]$} \\
$\tau_{\text {rad.P }}(\mathrm{ns})$ & 4.1 & {$[18]$} \\
$q_{\mathrm{P}}$ & 0.35 & {$[18]$} \\
$E_{\mathrm{P}}^{0}\left(\mathrm{~cm}^{-1}\right)$ & 727 & {$[17.18]$} \\
$x_{\mathrm{P}, \mathrm{h}}^{0}$ & 0.029 & eq. $(4)$ \\
& $\tau_{\mathrm{PN}}^{0}(\mathrm{~ms})$ & $2.15 \pm 0.1$ & this work \\
& $\sigma_{\mathrm{N}}\left(\lambda_{\mathrm{pr}}\right)\left(\mathrm{cm}^{2}\right)$ & $5.4 \times 10^{-18}$ & {$[18]$} \\
& $\sigma_{\mathrm{P}}\left(\lambda_{\mathrm{pr}}\right)\left(\mathrm{cm}^{2}\right)$ & $3.65 \times 10^{-16}$ & {$[18]$} \\
\hline
\end{tabular}

The ground-state $\mathrm{N}$-isomer and $\mathrm{P}$-isomer populations are governed by the pump light excitation dynamics and the ground-state $\mathrm{N}$ to $\mathrm{P}\left(k_{\mathrm{NP}}^{0}\right)$ and $\mathrm{P}$ to $\mathrm{N}\left(k_{\mathrm{PN}}^{0}\right)$ transfer rates. Assuming an Arrhenius type barrier crossing process, $k_{\mathrm{NP}}^{0}$ and $k_{\mathrm{PN}}^{0}$ are given by

$k_{\mathrm{NP}}^{0}=k_{0}^{0} \exp \left[-\left(E_{\mathrm{P}}^{0}+E_{\mathrm{A}}^{0}\right) / k_{\mathrm{B}} \vartheta\right]$,

$k_{\mathrm{PN}}^{0}=k_{0}^{0} \exp \left(-E_{\mathrm{A}}^{0} / k_{\mathrm{B}} \vartheta\right)$,

where $k_{\mathrm{B}}$ is the Boltzmann constant, $\vartheta$ the temperature, and $k_{0}^{0}$ the pre-exponential factor. Without excitation (thermal equilibrium) the ground-state population ratio is given by

$\frac{V_{4, \text { th }}}{V_{1, \text { th }}}=\frac{k_{\mathrm{NP}}^{0}}{k_{\mathrm{PN}}^{0}}=\exp \left(-E_{\mathrm{P}}^{0} / k_{\mathrm{B}} \vartheta\right)$,

where $N_{4, \text { th }}$ and $N_{1, \text { th }}$ are the thermal number densities of molecules in the $\mathrm{S}_{0} \mathrm{P}$-isomer and $\mathrm{N}$-isomer state, respectively. The thermal mole fraction of molecules in the $\mathrm{P}$-isomer ground state is

$x_{\mathrm{P}, \mathrm{th}}^{0}=\frac{N_{4, \mathrm{th}}}{N_{0}}=\frac{N_{4, \mathrm{th}}}{N_{1, \mathrm{th}}+N_{4, \mathrm{th}}}=\frac{\exp \left(-E_{\mathrm{P}}^{0} / k_{\mathrm{B}} \vartheta\right)}{1+\exp \left(-E_{\mathrm{P}}^{0} / k_{\mathrm{B}} \vartheta\right)}$,

where $N_{0}$ is the total number density of DODCI molecules.

The level populations caused by laser excitation are probed by transmission measurements of a weak He-Ne laser $\left(\lambda_{\mathrm{p}}=632.8 \mathrm{~nm}\right)$. The probe laser transmission is

$T_{\mathrm{pr}}=\exp \left\{-\left[\sigma_{\mathrm{N}}\left(\lambda_{\mathrm{pr}}\right) N_{1}+\sigma_{\mathrm{P}}\left(\lambda_{\mathrm{pr}}\right) N_{4}+\sigma_{\mathrm{N}}^{\mathrm{ex}}\left(\lambda_{\mathrm{pr}}\right) N_{2}+\sigma_{\mathrm{P}}^{\mathrm{ex}}\left(\lambda_{\mathrm{pr}}\right) N_{3}\right] l\right\}$,

where $\sigma_{\mathrm{N}}$ and $\sigma_{\mathrm{P}}$ are the apparent ground-state absorption cross sections while $\sigma_{\mathrm{N}}^{\mathrm{ex}}$ and $\sigma_{\mathrm{P}}^{\mathrm{ex}}$ are the excited-state absorption cross sections of the $\mathrm{N}$ and $\mathrm{P}$ isomers, respectively. $N_{1}$ and $N_{4}$ are the number densities of molecules in the $\mathrm{S}_{0} \mathrm{~N}$-isomer and $\mathrm{S}_{0} \mathrm{P}$-isomer state, respectively. $N_{2}$ is the number density of dye molecules in the $\mathrm{N}$ isomer $S_{1}$ state and $N_{3}$ is the number density of dye molecules in the P-isomer $S_{1}$ state. $l$ is the sample length. $\sigma_{\mathrm{N}}\left(\lambda_{\mathrm{pr}}\right)$ and $\sigma_{\mathrm{P}}\left(\lambda_{\mathrm{pr}}\right)$ have been separated previously by temperature-dependent long-wavelength absorption measurements $[17,18]$. The values are listed in table 1 . The total number density of DODCI molecules is $N_{0}=N_{1}+N_{2}+N_{3}+N_{4}$.

In our experiments the $S_{1}$-level populations remain negligibly small because the excitation intensities $I_{\mathrm{L}}$ are small compared to the saturation intensities $I_{N . S}^{1}=h \nu_{L} / \sigma_{N}\left(\lambda_{L}\right) \tau_{N}$ and $I_{\mathrm{P} . S}^{1}=h \nu_{L} / \sigma_{P}\left(\lambda_{L}\right) \tau_{P}$ of ground-state absorption bleaching which are in the $\mathrm{MW} / \mathrm{cm}^{2}$ region ( $h$ is the Planck constant) [20]. The neglect of the $S_{1}-$ state populations $N_{2}$ and $N_{3}$ leads to 


$$
\begin{aligned}
& T_{\mathrm{pr}}=\exp \left[-\sigma_{\mathrm{N}}\left(\lambda_{\mathrm{pr}}\right) N_{1} l-\sigma_{\mathrm{p}}\left(\lambda_{\mathrm{pr}}\right) N_{4} l\right]=\exp \left\{-\sigma_{\mathrm{N}}\left(\lambda_{\mathrm{pr}}\right) N_{0} l-\left[\sigma_{\mathrm{p}}\left(\lambda_{\mathrm{pr}}\right)-\sigma_{\mathrm{N}}\left(\lambda_{\mathrm{pr}}\right)\right] N_{4} l_{i}\right. \\
& \quad=\exp \left\{-N_{0} l\left\{\sigma_{\mathrm{N}}\left(\lambda_{\mathrm{pr}}\right)+\left[\sigma_{\mathrm{p}}\left(\lambda_{\mathrm{pr}}\right)-\sigma_{\mathrm{N}}\left(\lambda_{\mathrm{pr}}\right)\right] x_{\mathrm{p}}^{0}\right\}\right\} .
\end{aligned}
$$

The mole fraction of ground-state $P$ isomers is obtained by solving eq. ( $5 b)$ for $x_{P}^{0}$. The result is

$x_{\mathrm{P}}^{0}=\frac{N_{4}}{N_{0}}=\frac{\sigma_{\mathrm{N}}\left(\lambda_{\mathrm{pr}}\right)+\ln \left(T_{\mathrm{pr}}\right) / N_{0} l}{\sigma_{\mathrm{N}}\left(\lambda_{\mathrm{pr}}\right)-\sigma_{\mathrm{p}}\left(\lambda_{\mathrm{pr}}\right)}$.

In the experiments $T_{\mathrm{pr}}$ is measured as a function of the excitation laser intensity $I_{\mathrm{L}}$ at wavelength $\lambda_{\mathrm{L}}$ and $x_{\mathrm{P}}^{0}\left(I_{\mathrm{L}}\right.$, $\left.i_{L}\right)$ is calculated.

After switching-off the pump laser at time $t=0$ the P-isomer mole fraction $x_{\mathrm{P}}^{0}$ relaxes back to the thermal value $x_{P, t h}^{0}$ according to

$x_{\mathrm{P}}^{0}(t)=\left[x_{\mathrm{P}}^{0}(0)-x_{\mathrm{P}, \mathrm{h}}^{0}\right] f(t)+x_{\mathrm{P}, \mathrm{h}}^{0}$,

with the relaxation function

$f(t)=\exp \left(-k_{\mathrm{PN}}^{0} t\right)=\exp \left(-t / \tau_{\mathrm{PN}}^{0}\right)=\frac{x_{\mathrm{P}}^{0}(t)-x_{\mathrm{P}, \mathrm{h}}^{0}}{x_{\mathrm{P}}^{0}(0)-x_{\mathrm{P}, \mathrm{h}}^{0}}$,

where $\tau_{\mathrm{PN}}^{0}=\left(k_{\mathrm{PN}}^{0}\right)^{-1}$ is the ground-state $\mathrm{P}$-isomer to $\mathrm{N}$-isomer relaxation time. From the time-resolved probe laser transmission measurements the relaxation function

$f_{\mathrm{m}}(t)=\frac{\ln \left(T_{\mathrm{pr}, \mathrm{th}}\right)-\ln \left[T_{\mathrm{pr}}(t)\right]}{\ln \left(T_{\mathrm{pr}, \mathrm{h}}\right)-\ln \left[T_{\mathrm{pr}}(0)\right]}$

is derived. $T_{\mathrm{pr}, \mathrm{th}}$ is the probe laser transmission at thermal equilibrium. $f_{\mathrm{m}}(\mathrm{t})$ is identical to $f(t)$ as is seen by insertion of eq. (6) into eq. (8). The experimental function $f_{\mathrm{m}}(t)$ will be fitted by $\exp \left(-k_{\mathrm{PN}}^{0} t\right)$ with the fitting parameter $k_{\mathrm{PN}}^{0}$.

\section{Experimental}

The experimental setup for the determination of the mole fraction of $P$ isomers in the ground state is shown in fig. 2. The pump laser is either a cw argon ion laser (Spectra Physics model 171) or an argon ion laser pumped $\mathrm{cw}$ dye laser. The argon ion laser wavelength is selected with a prism $\left(\lambda_{\mathrm{L}}=488\right.$ and $\left.514 \mathrm{~nm}\right)$. The dyes rhodamine 110 in ethylene glycol [21] (wavelength range 550-570 $\mathrm{nm}$ ) and rhodamine 6G in ethylene glycol

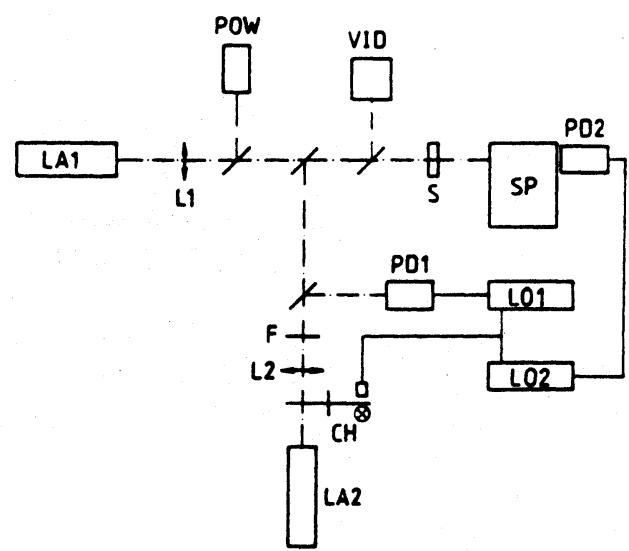

Fig. 2. Experimental setup for steady-state $S_{0} P$-isomer accumulation measurements. LA1, cw pump laser. LA2, cw He-Ne probe laser. L1, L2, lenses. F, filter. POW, power meter. VID, vidicon. S. DODCI sample in flowing cell. SP, spectrometer or dispersion prisms. PD1, PD2, photodetectors. CH, chopper. LO1, LO2, lockin amplifiers. 
$\left(\lambda_{L}=580-620 \mathrm{~nm}\right)$ are applied. The dye laser wavelength is tuned with a single-plate quartz birefringence filter (thickness $0.5 \mathrm{~mm}$ ).

For high excitation intensities the pump laser is focused slightly to the sample. The pump laser power $P_{\mathrm{L}}$ is detected with a power meter (Gentec model PS10 thermopile). The beam profiles and beam diameters of the pump and probe laser are monitored (in succession applying appropriate filters) with a standard vidicon (BuM Spectronik optical spectrum analyser ). The beam profiles are nearly Gaussian. The relation between the pump laser peak intensity $I_{\mathrm{OL}}$, the power $P_{\mathrm{L}}$, and the beam diameter $\Delta d_{\mathrm{L}}$ is

$I_{0 \mathrm{~L}}=I_{\mathrm{L}}(r=0)=4 \ln (2) P_{\mathrm{L}} / \pi\left(\Delta d_{\mathrm{L}}\right)^{2}$.

The beam diameter $\Delta d_{\mathrm{pr}}$ of the He-Ne probe laser is made approximately a factor of three smaller than the pump laser diameter.

The dye DODCI in ethylene glycol is circulated through a flowing dye cell (cell: Hellma type 134.070-QS; pump system: Lambda Physics dye circulator type FL40). The flow velocity is kept low $\left(v \approx 0.02 \mathrm{~ms}^{-1}\right)$ to avoid dye replacement within the ground-state P-isomer recovery time $\tau_{\mathrm{PN}}^{0}=\left(k_{\mathrm{PN}}^{0}\right)^{-1}$ (dye replacement time $\tau_{\text {rep }} \approx \Delta d$ ) $l: \approx 50 \mathrm{~ms}$ for $\Delta d_{\mathrm{L}} \approx 1 \mathrm{~mm}$ and $\tau_{\mathrm{PN}}^{0} \approx 2.15 \mathrm{~ms}$ at $25^{\circ} \mathrm{C}$. see below).

The He-Ne probe laser transmission is measured with two Si-photodetectors PD1 and PD2 (United Detector Technology type PIN 10D). For sensitive detection lock-in amplifiers are applied and the He-Ne laser is chopped. Behind the sample the probe laser is separated from the pump laser either by three dispersion prisms and an interference filter or a grating spectrometer.

The influence of intersystem crossing and triplet absorption is checked by sample excitation at $\lambda_{L}=514 \mathrm{~nm}$ and absorption probing at $\lambda_{\mathrm{pr}}=667 \mathrm{~nm}$ (triplet absorption has $T_{1}-T_{2}$ absorption peak at $\lambda \approx 667 \mathrm{~nm}$ [10]). A tungsten lamp filtered with an interference filter is used as probe light. At $\lambda_{\mathrm{pr}}=667 \mathrm{~nm}$ the N- and P-isomer long-wavelength singlet absorption at thermal equilibrium is very small $\left(\sigma(667 \mathrm{~nm}) \approx 2 \times 10^{-19} \mathrm{~cm}^{2}[18]\right.$, $\tau_{\mathrm{pr}}\left(667 \mathrm{~nm}, I_{\mathrm{L}} \rightarrow 0\right)=0.9988$ for the experimental DODCI concentration of $C=2.5 \times 10^{-5} \mathrm{~mol} / \mathrm{dm}^{3}$ and a sample length of $l=4 \mathrm{~mm})$. At $I_{0 \mathrm{~L}}=150 \mathrm{~W} / \mathrm{cm}^{2}$ the probe light transmission decreased to $T_{\mathrm{pr}}(667 \mathrm{~nm})=0.97 \pm 0.02$. The expected transmission due to P-isomer accumulation is $T_{\text {expected }}=0.97\left(\sigma_{\mathrm{P}}(667 \mathrm{~nm}) \approx 5 \times 10^{-18} \mathrm{~cm}^{2}[18]\right.$, $x_{\mathrm{P}}^{0}=0.69$, see fig. 5 below). Using a triplet absorption cross section of $\sigma_{\mathrm{T}}(667 \mathrm{~nm}) \approx 4.8 \times 10^{-16} \mathrm{~cm}^{2}[10]$, the mole fraction of DODCI molecules, $x_{\mathrm{T}}=N_{\mathrm{T}} / N_{0}$, transferred to the triplet state is estimated to be $x_{\mathrm{T}}\left(I_{\mathrm{OL}}=150\right.$ $\left.\mathrm{W} / \mathrm{cm}^{2}\right) \leqslant 0.007$. This fraction is neglected in the further analysis.

The probe laser transmission recovery after pump laser blocking is measured with a slightly changed experimental arrangement. The excitation wavelength is set to $\lambda_{L}=514 \mathrm{~nm}$ and the argon ion laser power is set to approximately $3.4 \mathrm{~W}$. The pump laser is chopped slowly and the transmitted cw probe laser signal (He-Ne laser) is detected with a photomultiplier (RCA type 1P28A instead of photodetector PD2) and recorded with a digital oscilloscope (Gould DSO 400).

\section{Results}

The cw probe laser transmission $T_{\mathrm{pr}}\left(\lambda_{\mathrm{pr}}=632.8 \mathrm{~nm}\right)$ versus pump laser peak intensity $I_{0 \mathrm{~L}}$ is displayed in figs. 3 and 4 for various excitation wavelengths $\lambda_{\mathrm{L}}$. The applied dye concentration is $C=2.5 \times 10^{-5} \mathrm{~mol} / \mathrm{dm}^{3}$ and the small-signal transmission is $T_{\mathrm{pr}, \mathrm{th}}=0.91$ ( sample length $l=4 \mathrm{~mm}, N_{0}=1.51 \times 10^{16} \mathrm{~cm}^{-3}$ ). Above $I_{0 \mathrm{~L}} \approx 0.1 \mathrm{~W} /$ $\mathrm{cm}^{2}$ the probe laser transmissions decrease and approach low transmission limits at high pump intensities. The curves present theoretical fits (see below).

From the experimental probe laser transmissions $T_{\mathrm{pr}}$ the mole fractions $x_{\mathrm{P}}^{0}$ of ground-state $\mathbf{P}$ isomers are calculated by use of eq. (6). The points in figs. 4 and 5 display the mole fractions $x_{\mathrm{P}}^{0}$ determined experimentally. The curves are theoretical fits ( see below). 


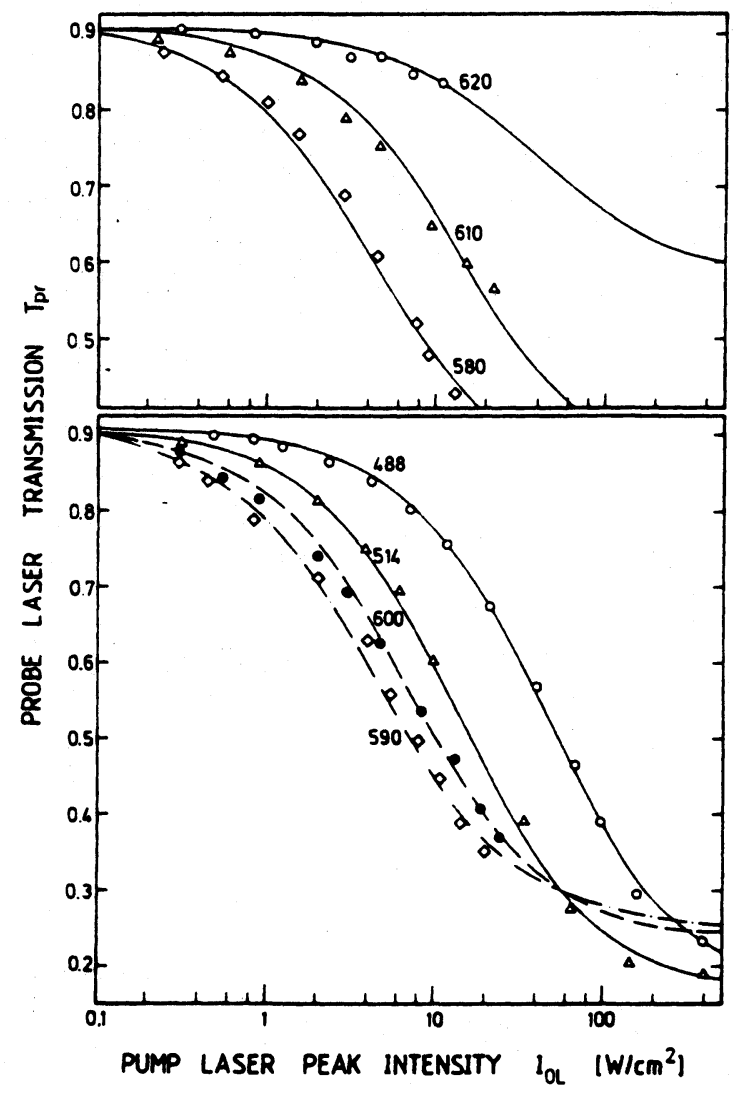

Fig. 3. Probe laser transmission $T_{\mathrm{pr}}\left(\lambda_{\mathrm{pr}}=632.8 \mathrm{~nm}\right)$ versus pump laser peak intensity $I_{O L}$ for various excitation wavelengths $\lambda_{L}$. The numbers indicate the pump laser wavelengths $\lambda_{L}$. The points are measured and the curves are fitted. The parameters used in the calculations are listed in table $I$ and displayed in fig. 6a. The inter-isomer transfer rates $\phi_{N P}^{\mathrm{tr}}$ and $\phi_{\mathrm{P}}^{\mathrm{W}} \mathrm{N}$ are fitted. The used values are plotted in fig. 8 (circles for $\phi_{\mathrm{PN}}^{\mathrm{tr}}$, dots for $\phi_{\mathrm{NP}}^{\mathrm{tt}}$ ).

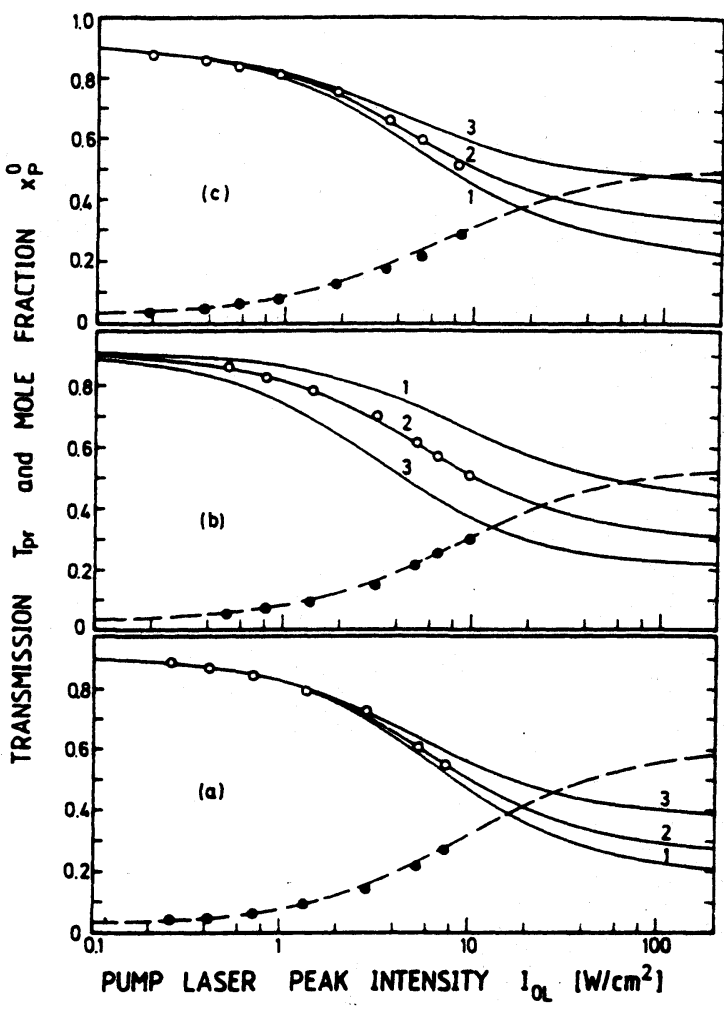

Fig. 4. Probe laser transmission $T_{\mathrm{pr}}\left(\lambda_{\mathrm{pr}}=632.8 \mathrm{~nm}\right)$ and steadystate accumulated $S_{0} P$-isomer mole fraction $x_{p}^{0}$ versus pump laser peak intensity $I_{0 L}$ for (a) $\lambda_{L}=550 \mathrm{~nm}$, (b) $\lambda_{L}=560 \mathrm{~nm}$, and (c) $\lambda_{\mathrm{L}}=570 \mathrm{~nm}$. Circles, experimental $T_{\text {pr }}$ points. Dots, experimen- tal $x_{p}^{0}$ data (eq. (6)). The curves are calculated (see legend of fig. 3 ). The dashed curves present the best fitting $x_{p}^{0}$ curves. The solid curves show $T_{\mathrm{pr}}$ curves with varying fit parameters: (a) $\sigma_{p}\left(i_{L}\right)$ is varied. The parameters are (1) $\sigma_{p}=3.5 \times 10^{-17} \mathrm{~cm}^{2}$, (2) $\sigma_{\mathrm{p}}=7 \times 10^{-17} \mathrm{~cm}^{2}$, (3) $\sigma_{\mathrm{p}}=1.4 \times 10^{-16} \mathrm{~cm}^{2}$ (b) $\phi_{\mathrm{NP}}$ is varied. The parameters are (1) $\phi_{N P}^{\text {LF }}=0.0185$, (2) $\phi_{N P}^{\mathrm{K}}=0.037$, (3) $\phi_{\mathrm{NP}}^{\mathrm{L}}=0.074$. (c) $\phi_{\mathrm{PN}}^{\mathrm{L}}$ is varied. The parameters are (1) $\phi_{\mathrm{PN}}^{\mathrm{HF}}=0.045$, (2) $\phi_{\mathrm{PN}}^{\mathrm{LF}}=0.09,(3) \phi_{\mathrm{PN}}^{\mathrm{H}}=0.18$.

The laser induced isomer redistribution alters the ground-state absorption ratio $\alpha_{P} / \alpha_{N}$ of $P$ isomers and $N$ isomers. The absorption coefficient ratio at the excitation wavelength $\lambda_{L}$ is

$\frac{\alpha_{\mathrm{P}}}{\alpha_{\mathrm{N}}}=\frac{x_{\mathrm{P}}^{0}\left(I_{\mathrm{OL}}\right) \sigma_{\mathrm{P}}\left(\lambda_{\mathrm{L}}\right)}{\left[1-x_{\mathrm{P}}^{0}\left(I_{0 \mathrm{~L}}\right)\right] \sigma_{\mathrm{N}}\left(\lambda_{\mathrm{L}}\right)}$

$\sigma_{N}\left(\lambda_{L}\right)$ and $\sigma_{P}\left(\lambda_{L}\right)$ are displayed in fig. $6 \mathrm{a}\left(\sigma_{N}\right.$ is redrawn from ref. [18], $\sigma_{\mathrm{P}}$ is taken from ref. [18] for $\lambda_{L}>550$ $\mathrm{nm}$ and determined in this work for $\lambda_{\mathrm{L}}=514$ and $488 \mathrm{~nm}$, see below ). $x_{\mathrm{P}}^{0}\left(I_{\mathrm{OL}}\right)$ is shown in figs. 4 and 5 for various excitation wavelengths $\lambda_{\mathrm{L}}$. For two examples, it is $\alpha_{\mathrm{P}} / \alpha_{\mathrm{N}}=0.12$ at $I_{0 \mathrm{~L}}=100 \mathrm{~W} / \mathrm{cm}^{2}$ and $\lambda_{\mathrm{L}}=488 \mathrm{~nm}$, and $\alpha_{\mathrm{P}} / \alpha_{\mathrm{N}}=3.1$ at $I_{\mathrm{OL}}=100 \mathrm{~W} / \mathrm{cm}^{2}$ and $\lambda_{\mathrm{L}}=620 \mathrm{~nm}$. In the long wavelength region the $\mathrm{P}$-isomer absorption coefficient dominates. Already at thermal equilibrium $\left(I_{0 L} \rightarrow 0\right)$ the absorption ratio is $\left(\alpha_{\mathrm{P}} / \alpha_{\mathrm{N}}\right)_{\mathrm{th}}=0.37$ at $\lambda=620$ 


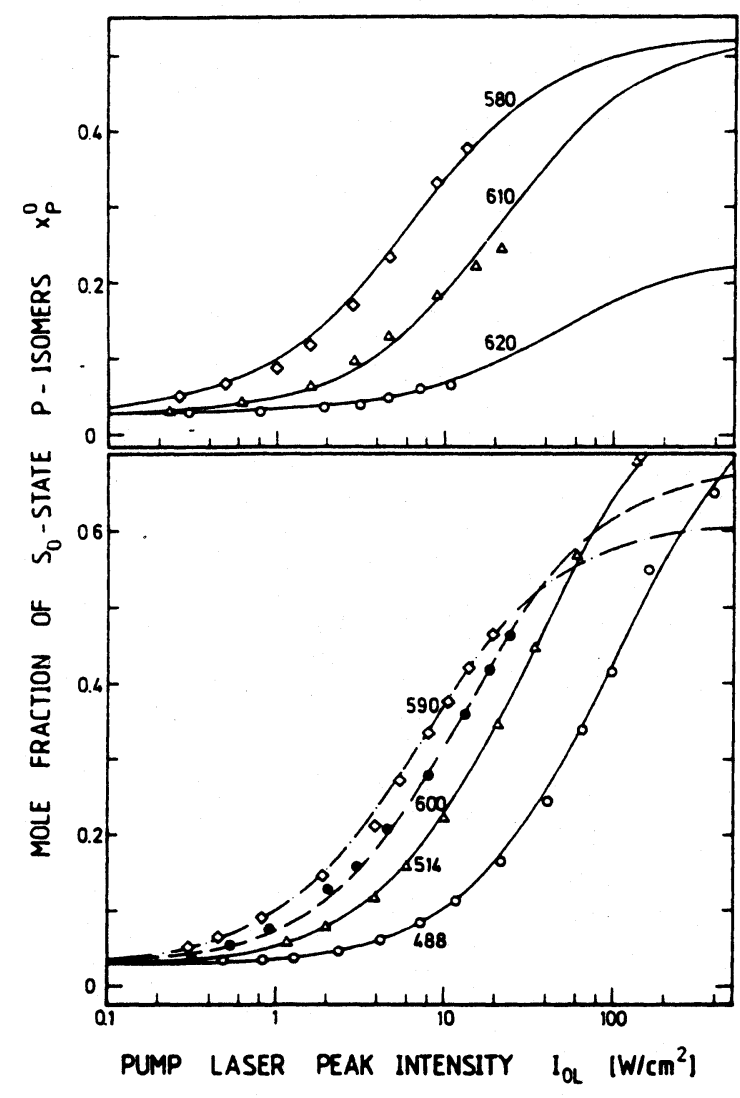

Fig. 5. Steady-state accumulated $S_{0}$-state $P$-isomer mole fraction $x_{\mathrm{P}}^{0}$ versus pump laser peak intensity $I_{0 L}$ for various excitation wavelengths $\lambda_{L}$. The numbers indicate the pump laser wavelengths. Experimental points according to eq. (6). Curves are calculated.

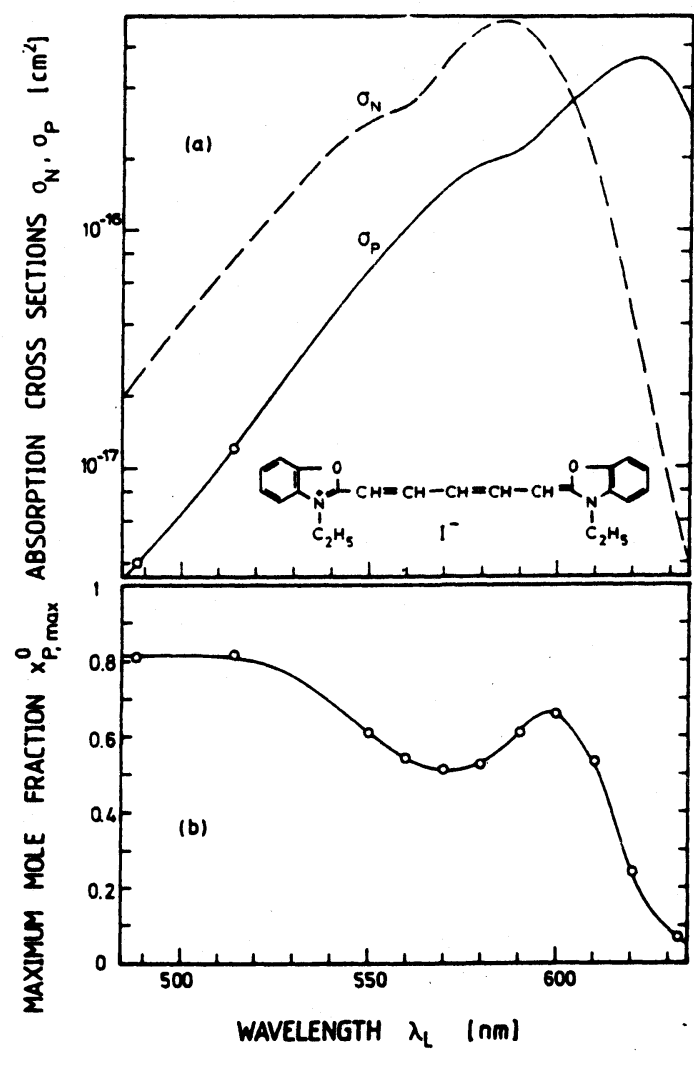

Fig. 6. (a) Absorption cross sections of DODCI in ethylene glycol at room temperature. Dashed curve, $\mathrm{N}$-isomer absorption cross sections, $\sigma_{N}\left(\lambda_{L}\right)$. Solid curve, $P$-isomer absorption cross sections, $\sigma_{P}\left(\lambda_{L}\right)$. Curves are taken from ref. $[18]$. Circles are $\sigma_{P}$ values obtained by fitting the $T_{p r}$ curves of fig. 3 to the measured probe pulse transmissions at high pump laser intensities. The structural formula of DODCl is included. (b) Calculated maximum $S_{0}$-state $P$-isomer mole fractions, $x_{P \text {,max }}^{0}$, at high pump laser intensity versus excitation wavelength. Curve is fitted through calculated $x_{\text {P.max }}^{0}$ points.

$\mathrm{nm}\left(x_{\mathrm{P}, \mathrm{th}}^{0}=0.029\right)$. The limiting ratios of $\alpha_{\mathrm{P}} / \alpha_{\mathrm{N}}$ for $I_{\mathrm{OL}} \rightarrow \infty$ are determined below (see fig. 8 ).

An example of the temporal probe laser transmission, $T_{\mathrm{pr}}(t)$, after pump laser blocking is displayed in fig. 7a. Corresponding relaxation function values $f_{\mathrm{m}}$ at discrete times are indicated by the circles in fig. $7 \mathrm{~b}$ (see eq. (9)). The exponential fit (straight line in fig. $7 \mathrm{~b}$ ) gives an $\mathrm{S}_{0}$-state $\mathrm{P}$ - to $\mathrm{N}$-isomer relaxation time of $\tau_{P N}^{0}=\left(k_{P N}^{0}\right)^{-1}=2.15 \pm 0.1 \mathrm{~ms}$. The temperature of the dye solution was $\vartheta=25^{\circ} \mathrm{C}$. In ref. [12] a value of $\tau_{\mathrm{PN}}^{0}=2.6$ $\mathrm{ms}$ is reported for $\theta=20^{\circ} \mathrm{C}$.

\section{Numerical simulations}

$\mathrm{N}$-isomer to $\mathrm{P}$-isomer and $\mathrm{P}$-isomer to $\mathrm{N}$-isomer transfer efficiencies and $\mathrm{P}$-isomer $\mathrm{S}_{\mathbf{0}}-\mathrm{S}_{\mathbf{1}}$ absorption cross sections are determined by numerical simulations. 


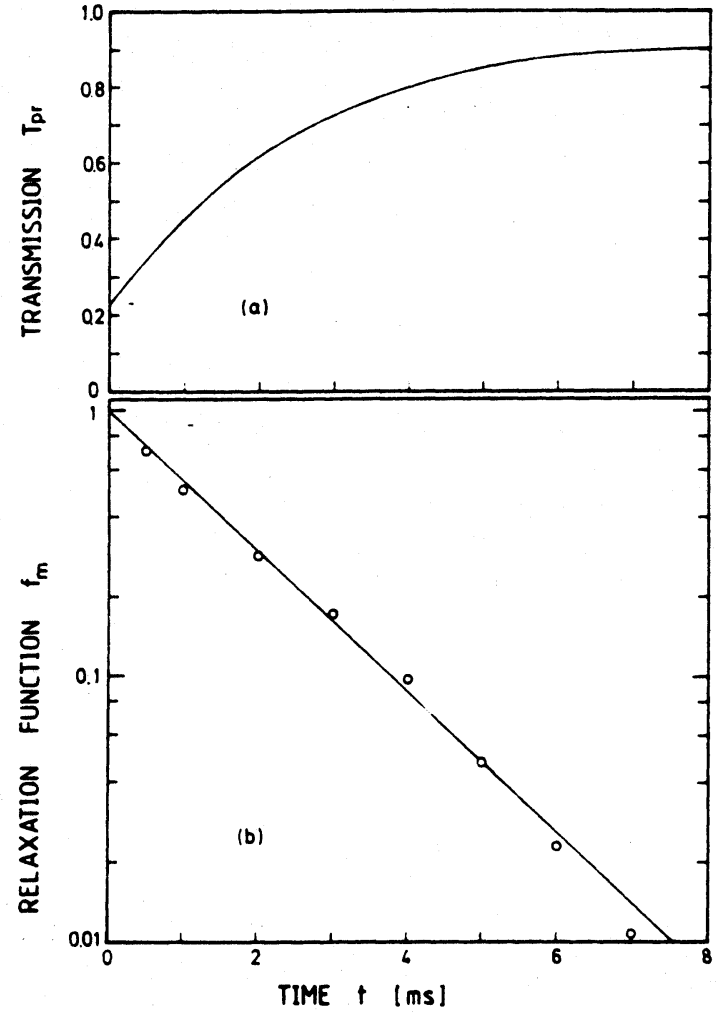

Fig. 7. Determination of ground-state P-isomer thermalization time $\tau_{\mathrm{pN}}^{0}$ by measurements of probe pulse transmission $T_{\mathrm{pr}}$ versus time after pump laser blocking (a). From the slope of the relaxation function $f_{\mathrm{m}}(t)(\mathrm{b})$ a relaxation time of $\tau_{\mathrm{PN}}^{0}=2.15 \pm 0.1$ $\mathrm{ms}$ is determined. The circles indicate the $f_{\mathrm{m}}(t)$ values obtained from the $T_{\mathrm{pr}}(t)$ curve at discrete times. Excitation: $\lambda_{\mathrm{L}}=514 \mathrm{~nm}$, $P_{\mathrm{L}}=3.4 \mathrm{~W}$.

\subsection{Steady-state theory}

The model of fig. 1 leads to the following rate equation system:

$\partial N_{1} / \partial t=-\left(\sigma_{N} I_{L} / h \nu_{\mathrm{L}}\right) N_{1}+k_{\mathrm{N}} N_{2}+k_{\mathrm{PN}}^{0} N_{4}-k_{\mathrm{NP}}^{0} N_{1}+k_{\mathrm{PN}}^{10} N_{3}$,

$\partial N_{2} / \partial t=\left(\sigma_{\mathrm{N}} I_{\mathrm{L}} / h \nu_{\mathrm{L}}\right) N_{1}-k_{\mathrm{N}} N_{2}-k_{\mathrm{NP}}^{1} N_{2}+k_{\mathrm{PN}}^{1} N_{3}-k_{\mathrm{NP}}^{10} N_{2}$,

$\partial N_{3} / \partial t=\left(\sigma_{\mathrm{P}} I_{\mathrm{L}} / h \nu_{\mathrm{L}}\right) N_{4}-k_{\mathrm{P}} N_{3}+k_{\mathrm{NP}}^{1} N_{2}-k_{\mathrm{PN}}^{1} N_{3}-k_{\mathrm{PN}}^{10} N_{3}$,

$\partial N_{4} / \partial t=-\left(\sigma_{\mathrm{P}} I_{\mathrm{L}} / h \nu_{\mathrm{L}}\right) N_{4}+k_{\mathrm{P}} N_{3}+k_{\mathrm{NP}}^{0} N_{1}-k_{\mathrm{PN}}^{0} N_{4}+k_{\mathrm{NP}}^{10} N_{2}$.

The total number density of DODCI molecules is $N_{0}=N_{1}+N_{2}+N_{3}+N_{4}$.

The cw pump laser excitation leads to a steady-state level population, i.e. $\partial N_{i} / \partial t=0$ where $i=1,2,3,4$, and eqs. (12a $)-(12 d)$ reduce to the following matrix equation:

$\left(\begin{array}{ccc}k_{N} & k_{\mathrm{PN}}^{10} & k_{\mathrm{PN}}^{0} \\ k_{\mathrm{N}}+k_{\mathrm{NP}}^{1}+k_{\mathrm{NP}}^{10} & -k_{\mathrm{PN}}^{1} & 0 \\ k_{\mathrm{NP}}^{10} & -k_{\mathrm{P}}^{10} & \sigma_{\mathrm{P}} I_{\mathrm{L}} / h \nu_{\mathrm{L}}+k_{\mathrm{PN}}^{0}\end{array}\right)\left(\begin{array}{c}N_{2} \\ N_{3} \\ N_{4}\end{array}\right)=\left(\begin{array}{c}\sigma_{\mathrm{N}} I_{\mathrm{L}} / h \nu_{\mathrm{L}}+k_{\mathrm{NP}}^{0} \\ \sigma_{\mathrm{N}} I_{\mathrm{L}} / h \nu_{\mathrm{L}} \\ k_{\mathrm{NP}}^{0}\end{array}\right) N_{1}$.

The Gaussian elimination procedure (iterative transfer to triangular matrix shape) [22] gives after some arithmetic rearrangements 


$$
\begin{aligned}
V_{4} & =\left\{\left(k_{\mathrm{NP}}^{10} k_{\mathrm{PN}}^{10}+k_{\mathrm{N}} k_{\mathrm{P}}\right)\left[\left(k_{\mathrm{N}}+k_{\mathrm{NP}}^{1}+k_{\mathrm{NP}}^{10}\right)\left(\sigma_{\mathrm{N}} I_{\mathrm{L}} / h \nu_{\mathrm{L}}+k_{\mathrm{NP}}^{0}\right)-k_{\mathrm{N}} \sigma_{\mathrm{N}} I_{\mathrm{L}} / h \nu_{\mathrm{L}}\right]\right. \\
& \left.+\left[-k_{\mathrm{NP}}^{10}\left(\sigma_{\mathrm{N}} I_{\mathrm{L}} / h \nu_{\mathrm{L}}+k_{\mathrm{NP}}^{0}\right)+k_{\mathrm{N}} k_{\mathrm{NP}}^{0}\right]\left[k_{\mathrm{N}} k_{\mathrm{PN}}^{1}+\left(k_{\mathrm{N}}+k_{\mathrm{NP}}^{1}+k_{\mathrm{NP}}^{10}\right) k_{\mathrm{PN}}^{10}\right]\right\} \\
& \times\left\{\left(k_{\mathrm{NP}}^{10} k_{\mathrm{PN}}^{10}+k_{\mathrm{N}} k_{\mathrm{P}}\right)\left(k_{\mathrm{N}}+k_{\mathrm{NP}}^{1}+k_{\mathrm{PN}}^{10}\right) k_{\mathrm{PN}}^{0}\right. \\
& \left.\left.+\left[-k_{\mathrm{NP}}^{10} k_{\mathrm{PN}}^{0}+k_{\mathrm{N}}\left(\sigma_{\mathrm{P}} I_{\mathrm{L}} / h \nu_{\mathrm{L}}+k_{\mathrm{PN}}^{0}\right)\right]\left[k_{\mathrm{N}} k_{\mathrm{PN}}^{1}+\left(k_{\mathrm{N}}+k_{\mathrm{NP}}^{1}+k_{\mathrm{NP}}^{10}\right) k_{\mathrm{PN}}^{10}\right]\right\}\right\}^{-1} N_{1}, \\
N_{3} & =\frac{\left[\left(k_{\mathrm{N}}+k_{\mathrm{NP}}^{1}+k_{\mathrm{NP}}^{10}\right)\left(\sigma_{\mathrm{N}} I_{\mathrm{L}} / h \nu_{\mathrm{L}}+k_{\mathrm{NP}}^{0}\right)-k_{\mathrm{N}} \sigma_{\mathrm{N}} I_{\mathrm{L}} / h \nu_{\mathrm{L}}\right] N_{1}-\left(k_{\mathrm{N}}+k_{\mathrm{NP}}^{1}+k_{\mathrm{NP}}^{10}\right) k_{\mathrm{PN}}^{0} N_{4}}{\left(k_{\mathrm{N}}+k_{\mathrm{NP}}^{1}+k_{\mathrm{NP}}^{10}\right) k_{\mathrm{PN}}^{10}+k_{\mathrm{N}} k_{\mathrm{PN}}^{1}}, \\
N_{2} & =\frac{\left(\sigma_{\mathrm{N}} I_{\mathrm{L}} / h \nu_{\mathrm{L}}+k_{\mathrm{NP}}^{0}\right) N_{1}-k_{\mathrm{PN}}^{10} N_{3}-k_{\mathrm{PN}}^{0} N_{4}}{k_{\mathrm{N}}}, \\
N_{1} & =N_{0}-N_{2}-N_{3}-N_{4} .
\end{aligned}
$$

Without excitation, i.e. $I_{\mathrm{L}} \rightarrow 0$, eqs. $(14 \mathrm{a})-(14 \mathrm{~d})$ reduce to

$$
\begin{aligned}
& N_{4}=\frac{k_{\mathrm{NP}}^{0}}{k_{\mathrm{PN}}^{0}}=\exp \left(-E_{\mathrm{P}}^{0} / k_{\mathrm{B}} \vartheta\right) N_{1}=\frac{\exp \left(-E_{\mathrm{P}}^{0} / k_{\mathrm{B}} \vartheta\right)}{1+\exp \left(-E_{\mathrm{P}}^{0} / k_{\mathrm{B}} \vartheta\right)} N_{0}, \\
& N_{3}=0, \\
& N_{4}=0, \\
& N_{1}=N_{0}-N_{4}=\left[1+\exp \left(-E_{\mathrm{P}}^{0} / k_{\mathrm{B}} \vartheta\right)\right]^{-1} N_{0} .
\end{aligned}
$$

Eq. ( $15 a$ ) is equal to eq. ( 3 ).

In the following two limiting cases are considered which simplify eqs. (14a)-(14d).

(i) Neglect of $\mathrm{S}_{1}-\mathrm{S}_{0}$ inter-isomer relaxation, i.e. $k_{\mathrm{PN}}^{10}=k_{\mathrm{NP}}^{10}=0$. Only the $\mathrm{S}_{1}$-state inter-isomer transfer is considered. Eqs. (14a) $-(14 d)$ reduce to

$$
\begin{aligned}
& N_{4}=\frac{k_{\mathrm{P}}\left[\left(k_{\mathrm{N}}+k_{\mathrm{NP}}^{1}\right) k_{\mathrm{NP}}^{0}+k_{\mathrm{NP}}^{1} \sigma_{\mathrm{N}} I_{\mathrm{L}} / h \nu_{\mathrm{L}}\right]+k_{\mathrm{N}} k_{\mathrm{NP}}^{0} k_{\mathrm{PN}}^{1}}{k_{\mathrm{P}}\left(k_{\mathrm{N}}+k_{\mathrm{NP}}^{1}\right) k_{\mathrm{PN}}^{0}+k_{\mathrm{N}} k_{\mathrm{PN}}^{0} k_{\mathrm{PN}}^{1}+k_{\mathrm{N}} k_{\mathrm{PN}}^{1} \sigma_{\mathrm{P}} I_{\mathrm{L}} / h \nu_{\mathrm{L}}} N_{1}, \\
& v_{3}=\frac{\left(k_{\mathrm{NP}}^{1} \sigma_{\mathrm{N}} I_{\mathrm{L}} / h \nu_{\mathrm{L}}+k_{\mathrm{NP}}^{1} k_{\mathrm{NP}}^{0}\right) N_{1}-\left(k_{\mathrm{N}}+k_{\mathrm{NP}}^{1}\right) k_{\mathrm{PN}}^{0} N_{4}}{k_{\mathrm{N}} k_{\mathrm{PN}}^{1}}, \\
& V_{2}=\frac{\left(\sigma_{\mathrm{N}} I_{\mathrm{L}} / h \nu_{\mathrm{L}}+k_{\mathrm{NP}}^{0}\right) N_{1}-k_{\mathrm{PN}}^{0} N_{4}}{k_{\mathrm{N}}}, \\
& V_{1}=N_{0}-N_{2}-N_{3}-N_{4} .
\end{aligned}
$$

(ii) Neglect of $\mathrm{S}_{1}$-state inter-isomer transfer, i.e. $k_{\mathrm{NP}}^{1}=k_{\mathrm{PN}}^{1}=0$. Only the $\mathrm{S}_{1}-\mathrm{S}_{0}$ inter-isomer relaxation is considered. Eqs. (14a) $-(14 d)$ reduce to

$$
\begin{aligned}
N_{4} & =\left\{\left(k_{\mathrm{NP}}^{10} k_{\mathrm{PN}}^{10}+k_{\mathrm{N}} k_{\mathrm{P}}\right)\left[k_{\mathrm{NP}}^{10} \sigma_{\mathrm{N}} I_{\mathrm{L}} / h \nu_{\mathrm{L}}+\left(k_{\mathrm{N}}+k_{\mathrm{NP}}^{10}\right) k_{\mathrm{NP}}^{0}\right]\right. \\
& \left.+\left[-k_{\mathrm{NP}}^{10}\left(\sigma_{\mathrm{N}} I_{\mathrm{L}} / h \nu_{\mathrm{L}}+k_{\mathrm{NP}}^{0}\right)+k_{\mathrm{N}} k_{\mathrm{NP}}^{0}\right]\left(k_{\mathrm{N}}+k_{\mathrm{NP}}^{10}\right) k_{\mathrm{PN}}^{10}\right\}\left\{\left(k_{\mathrm{NP}}^{10} k_{\mathrm{PN}}^{10}+k_{\mathrm{N}} k_{\mathrm{P}}\right)\left(k_{\mathrm{N}}+k_{\mathrm{NP}}^{10}\right) k_{\mathrm{PN}}^{0}\right. \\
& \left.+\left[-k_{\mathrm{NP}}^{10} k_{\mathrm{PN}}^{0}+k_{\mathrm{N}}\left(\sigma_{\mathrm{P}} I_{\mathrm{L}} / h \nu_{\mathrm{L}}+k_{\mathrm{PN}}^{0}\right)\right]\left(k_{\mathrm{N}}+k_{\mathrm{NP}}^{10}\right) k_{\mathrm{PN}}^{10}\right\}^{-1} N_{1}, \\
\dot{V}_{3} & =\frac{k_{\mathrm{NP}}^{10}\left(\sigma_{\mathrm{N}} I_{\mathrm{L}} / h \nu_{\mathrm{L}}+k_{\mathrm{NP}}^{0}\right) N_{1}-\left(k_{\mathrm{N}}+k_{\mathrm{NP}}^{10}\right) k_{\mathrm{PN}}^{0} N_{4}}{\left(k_{\mathrm{N}}+k_{\mathrm{NP}}^{10}\right) k_{\mathrm{PN}}^{10}} \\
N_{2} & =\frac{\left(\sigma_{\mathrm{N}} I_{\mathrm{L}} / h \nu_{\mathrm{L}}+k_{\mathrm{NP}}^{0}\right) N_{1}-k_{\mathrm{PN}}^{10} N_{3}-k_{\mathrm{PN}}^{0} N_{4}}{k_{\mathrm{N}}} \\
N_{1} & =N_{0}-N_{2}-N_{3}-N_{4} .
\end{aligned}
$$


Using the approximations $k_{\mathrm{NP}}^{10} \ll k_{\mathrm{N}}$ and $k_{\mathrm{PN}}^{10} \ll k_{\mathrm{P}}$ which are fulfilled experimentally (see below) eqs. (17a)(17d) and (16a)-(16d) become approximately equal if $k_{\mathrm{PN}}^{10}$ and $k_{\mathrm{PN}}^{1}$ are replaced by $k_{\mathrm{PN}}^{\mathrm{tr}}$, and $k_{\mathrm{NP}}^{10}$ and $k_{\mathrm{NP}}^{1}$ are replaced by $k_{\mathrm{NP}}^{\mathrm{tr}}$. For this situation of small inter-isomer transfer, $k_{\mathrm{NP}}^{\mathrm{tr}}$ and $k_{\mathrm{PN}}^{\mathrm{tr}}$, compared to $\mathrm{S}_{1}-\mathrm{S}_{0}$ intraisomer relaxation, $k_{\mathrm{N}}$ and $k_{\mathrm{p}}$, the following equation system may be written:

$N_{4}=\frac{k_{\mathrm{P}}\left[\left(k_{\mathrm{N}}+k_{\mathrm{NP}}^{\mathrm{tr}}\right) k_{\mathrm{NP}}^{0}+k_{\mathrm{NP}}^{\mathrm{tr}} \sigma_{\mathrm{N}} I_{\mathrm{L}} / h \nu_{\mathrm{L}}\right]+k_{\mathrm{N}} k_{\mathrm{NP}}^{0} k_{\mathrm{PN}}^{\mathrm{tr}}}{k_{\mathrm{P}}\left(k_{\mathrm{N}}+k_{\mathrm{NP}}^{\mathrm{tr}}\right) k_{\mathrm{PN}}^{0}+k_{\mathrm{N}} k_{\mathrm{PN}}^{0} k_{\mathrm{PN}}^{\mathrm{tr}}+k_{\mathrm{N}} k_{\mathrm{PN}}^{\mathrm{tr}} \sigma_{\mathrm{P}} I_{\mathrm{L}} / h \nu_{\mathrm{L}}} N_{1}$,

$N_{3}=\frac{\left(k_{\mathrm{NP}}^{\mathrm{tr}} \sigma_{\mathrm{N}} I_{\mathrm{L}} / h \nu_{\mathrm{L}}+k_{\mathrm{NP}}^{\mathrm{tr}} k_{\mathrm{NP}}^{0}\right) N_{1}-\left(k_{\mathrm{N}}+k_{\mathrm{NP}}^{\mathrm{tr}}\right) k_{\mathrm{PN}}^{0} N_{4}}{k_{\mathrm{N}} k_{\mathrm{PN}}^{\mathrm{tr}}}$,

$N_{2}=\frac{\left(\sigma_{\mathrm{N}} I_{\mathrm{L}} / h \nu_{\mathrm{L}}+k_{\mathrm{NP}}^{0}\right) N_{1}-k_{\mathrm{PN}}^{0} N_{4}}{k_{\mathrm{N}}}$,

$N_{1}=N_{0}-N_{2}-N_{3}-N_{4}$.

These equations are used in our numerical simulations.

Instead of the transfer rates $k_{\mathrm{NP}}^{\mathrm{tr}}$ and $k_{\mathrm{PN}}^{\mathrm{tr}}$ the transfer efficiencies $\phi_{\mathrm{NP}}^{\mathrm{tr}}$ and $\phi_{\mathrm{PN}}^{\mathrm{tr}}$ may be determined. They are related to the transfer rates and the fluorescence quantum efficiencies by [18]

$\phi_{\mathrm{NP}}^{\mathrm{tr}}=\frac{k_{\mathrm{NP}}^{\mathrm{tr}}}{k_{\text {tot.N }}}=\frac{k_{\mathrm{NP}}^{\mathrm{tr}}}{k_{\mathrm{rad} . \mathrm{N}}} q_{\mathrm{N}}=k_{\mathrm{NP}}^{\mathrm{tr}} \tau_{\mathrm{rad}, \mathrm{N}} q_{\mathrm{N}}=k_{\mathrm{NP}}^{\mathrm{tr}} \tau_{\mathrm{N}}$

and $i$

$\phi_{\mathrm{PN}}^{\mathrm{tr}}=\frac{k_{\mathrm{PN}}^{\mathrm{tr}}}{k_{\text {rot, } \mathrm{P}}}=\frac{k_{\mathrm{PN}}^{\mathrm{tr}}}{k_{\text {rad, } \mathrm{P}}} q_{\mathrm{P}}=k_{\mathrm{PN}}^{\mathrm{tr}} \tau_{\text {rad, } \mathrm{P}} q_{\mathrm{P}}=k_{\mathrm{PN}}^{\mathrm{tr}} \tau_{\mathrm{P}}$.

$k_{\mathrm{N}}$ and $k_{\mathrm{P}}$ are given by

$k_{\mathrm{N}}=k_{\text {tot. }}-k_{\mathrm{NP}}^{\mathrm{\& r}}=\left(1-\phi_{\mathrm{NP}}^{\mathrm{\&}}\right) / \tau_{\mathrm{N}}$,

$k_{\mathrm{P}}=k_{\text {lot.P }}-k_{\mathrm{PN}}^{\mathrm{tr}}=\left(1-\phi_{\mathrm{PN}}^{\mathrm{tr}}\right) / \tau_{\mathrm{P}}$.

In the case of very strong excitation, the intensity-dependent terms in the numerator and in the denominator of eq. (18a ) dominate and the level populations reduce to

$N_{4}=\frac{k_{\mathrm{P}}}{k_{\mathrm{N}}} \frac{k_{\mathrm{NP}}^{\mathrm{tr}}}{k_{\mathrm{PN}}^{\mathrm{tr}}} \frac{\sigma_{\mathrm{N}}}{\sigma_{\mathrm{P}}} N_{1}=\frac{\left(1-\phi_{\mathrm{PN}}^{\mathrm{r}}\right) \phi_{\mathrm{NP}}^{\mathrm{r}} \sigma_{\mathrm{N}}}{\left(1-\phi_{\mathrm{NP}}^{\mathrm{tr}}\right) \phi_{\mathrm{PN}}^{\mathrm{tr}} \sigma_{\mathrm{P}}} N_{1}$,

$N_{3}=\frac{k_{\mathrm{NP}}^{\mathrm{tr}} \sigma_{\mathrm{N}} I_{\mathrm{L}}}{k_{\mathrm{PN}}^{\mathrm{tr}} k_{\mathrm{N}} h \nu_{\mathrm{L}}} N_{1}-\frac{\left(k_{\mathrm{N}}+k_{\mathrm{NP}}^{\mathrm{tr}}\right) k_{\mathrm{PN}}^{0}}{k_{\mathrm{N}} k_{\mathrm{PN}}^{\mathrm{tr}}} N_{4}=\frac{\phi_{\mathrm{NP}}^{\mathrm{tr}} \tau_{\mathrm{P}} \sigma_{\mathrm{N}} I_{\mathrm{L}}}{\phi_{\mathrm{PN}}^{\mathrm{tr}} h \nu_{\mathrm{L}}} N_{1}-\frac{\tau_{\mathrm{P}}}{\left(1-\phi_{\mathrm{NP}}^{\mathrm{tr}}\right) \phi_{\mathrm{PN}}^{\mathrm{tr}} \tau_{\mathrm{PN}}^{0}} N_{4}$,

$N_{2}=\frac{\sigma_{\mathrm{N}} I_{\mathrm{L}}}{k_{\mathrm{N}} h \nu_{\mathrm{L}}} N_{1}-\frac{k_{\mathrm{PN}}^{0}}{k_{\mathrm{N}}} N_{4}=\frac{\tau_{\mathrm{N}} \sigma_{\mathrm{N}} I_{\mathrm{L}}}{\left(1-\phi_{\mathrm{NP}}^{\mathrm{k}}\right) h \nu_{\mathrm{L}}} N_{1}-\frac{\tau_{\mathrm{N}}}{\left(1-\phi_{\mathrm{NP}}^{\mathrm{k}}\right) \tau_{\mathrm{PN}}^{0}} N_{4}$,

$N_{1}=N_{0}-N_{2}-N_{3}-N_{4}$.

Neglecting the weak $S_{1}$-level populations, eqs. (20a) and (20d) give

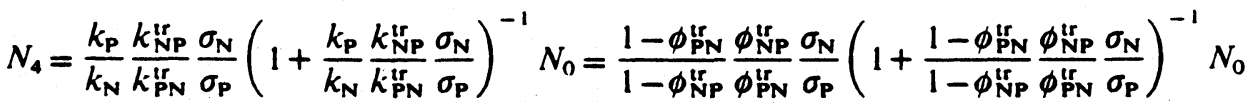

and

$N_{1}=\left(1+\frac{k_{\mathrm{P}}}{k_{\mathrm{N}}} \frac{k_{\mathrm{NP}}^{\mathrm{L}}}{k_{\mathrm{PN}}^{\mathrm{H}}} \frac{\sigma_{\mathrm{N}}}{\sigma_{\mathrm{P}}}\right)^{-1} N_{0}=\left(1+\frac{1-\phi_{\mathrm{PN}}^{\mathrm{L}}}{1-\phi_{\mathrm{NP}}^{\mathrm{H}}} \frac{\phi_{\mathrm{NP}}^{\mathrm{L}}}{\phi_{\mathrm{PN}}^{\mathrm{L}}} \frac{\sigma_{\mathrm{N}}}{\sigma_{\mathrm{P}}}\right)^{-1} N_{0}$

For the ground-state $\mathrm{P}$-isomer accumulation process an absolute accumulation saturation intensity, $I_{\mathbf{S}, \mathbf{P}}^{0.4 b}$, 
may be defined by the pump laser intensity where the steady state level populations $N_{4}$ and $N_{1}$ are equal. This condition leads after some rearrangements of eq. (18a) to

$I_{S . P}^{0 . a b s}=\frac{h \nu_{\mathrm{L}} k_{\mathrm{PN}}^{0}\left[1-\exp \left(-E_{\mathrm{P}}^{0} / k_{\mathrm{B}} \vartheta\right)\right]\left(1-\phi_{\mathrm{NP}}^{\mathrm{tr}} \phi_{\mathrm{P} N}^{\mathrm{tr}}\right)}{\left(1-\phi_{\mathrm{PN}}^{\mathrm{tr}}\right) \phi_{\mathrm{NP}}^{\mathrm{tr}} \sigma_{\mathrm{N}}-\left(1-\phi_{\mathrm{NP}}^{\mathrm{tr}}\right) \phi_{\mathrm{P} N}^{\mathrm{tr}} \sigma_{\mathrm{P}}}$.

If $I_{\mathrm{S} . \mathrm{P}}^{0 . a b s}<0$ (i.e. $\left(1-\phi_{\mathrm{PN}}^{\mathrm{tr}}\right) \phi_{\mathrm{NP}}^{\mathrm{tr}} \sigma_{\mathrm{N}}<\left(1-\phi_{\mathrm{NP}}^{\mathrm{tr}}\right) \phi_{\mathrm{PN}}^{\mathrm{tr}} \sigma_{\mathrm{P}}$ ) an accumulation of $\mathrm{P}$ isomers in the $\mathrm{S}_{0}$ state up to $x_{\mathrm{P}}^{0}=0.5$ is not possible.

A relative accumulation saturation intensity, $I_{S . P}^{0, \text { rel }}$, may be defined by the pump laser intensity where $N_{4} / N_{1}$ (eq. (18a)) is equal to one half of the limiting $N_{4} / N_{0}$ ratio at high intensities (eq. (21a)). This condition leads to

$I_{\mathrm{S} . \mathrm{P}}^{0, \mathrm{rel}}=\frac{h \nu_{\mathrm{L}} k_{\mathrm{PN}}^{0}\left(1-\phi_{\mathrm{NP}}^{\mathrm{tr}} \phi_{\mathrm{PN}}^{\mathrm{tr}}\right)\left[1-\exp \left(-E_{\mathrm{P}}^{0} / k_{\mathrm{B}} \vartheta\right)\right]}{\sigma_{\mathrm{P}} \phi_{\mathrm{PN}}^{\mathrm{tr}}\left[1-\phi_{\mathrm{NP}}^{\mathrm{r}}+2\left(1-\phi_{\mathrm{PN}}^{\mathrm{tr}}\right) \phi_{\mathrm{NP}}^{\mathrm{tr}} \sigma_{\mathrm{N}} / \phi_{\mathrm{PN}}^{\mathrm{tr}} \sigma_{\mathrm{P}}\right]} \approx \frac{h \nu_{\mathrm{L}} k_{\mathrm{PN}}^{0}}{\sigma_{\mathrm{P}} \phi_{\mathrm{PN}}^{\mathrm{tr}}}$

\subsection{Parameter determination}

Most DODCI parameters entering eqs. (18a)-(18d) and (19a), (19b) have been determined in two previous papers $[17,18]$. They are listed in table 1 . The $\sigma_{N}\left(\lambda_{L}\right)$ and $\sigma_{P}\left(\lambda_{L}\right)$ curves are shown in fig. $6 a$. $k_{\mathrm{PN}}^{0}$ has been measured in this work (fig. 7, see also ref. [12]) and is included in table 1.

The unknown transfer efficiencies $\phi_{\mathrm{NP}}^{\mathrm{tr}}$ and $\phi_{\mathrm{PN}}^{\mathrm{tr}}$ are determined by fitting the theoretical calculations to the experimental $T_{\mathrm{pr}}$ (eq. (5)) and $x_{\mathrm{p}}^{0}$ data (eq. (6)) (see curves in figs. 3-6). The fitted $\phi_{\mathrm{NP}}^{\mathrm{tr}}$ and $\phi_{\mathrm{PN}}^{\mathrm{t}}$ parameters depend on the excitation wavelength. They are displayed in fig. 8 . The transfer efficiencies are very low in the

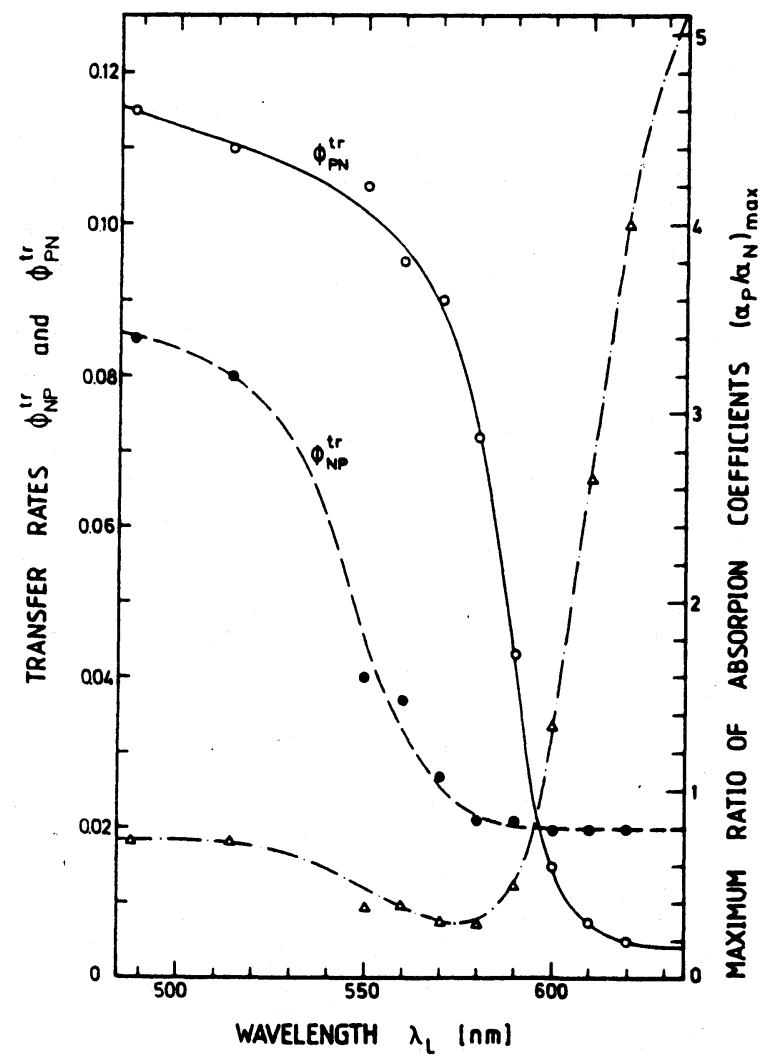

Fig. 8. Fitted photo-induced inter-isomer transfer efficiencies $\phi$ If (circles and solid curve) and $\phi$ isp (dots and dashed curve) versus pump laser wavelength $\lambda_{L}$. The ratio of the absorptior coefficients $\alpha_{p} / \alpha_{N}$ at high pump pulse intensities is included (triangles and dash-dotted curve). 
case of zero-vibration $S_{0}-S_{1}$ excitation $\left(\lambda_{L} \geqslant 590 \mathrm{~nm}\right.$ for $N$-isomer excitation, $\phi_{\mathrm{NP}}^{\mathrm{ir}}\left(\lambda_{\mathrm{L}} \geqslant 590 \mathrm{~nm}\right) \approx 0.02: \lambda_{\mathrm{L}} \geqslant 620$ $\mathrm{nm}$ for P-isomer excitation, $\left.\phi_{\mathrm{PN}}^{\mathrm{tr}}\left(\lambda_{\mathrm{L}} \geqslant 620 \mathrm{~nm}\right) \approx 0.005\right)$. They increase with excess energy in the $S_{1}$ state. For all experimental excitation wavelengths, it was $\phi_{\mathrm{NP}}^{\mathrm{tr}}<0.09$ and $\phi_{\mathrm{PN}}^{\mathrm{tr}}<0.12$. Therefore the simplification of eqs. $(16 a)-(16 d)$ and $(17 a)-(17 d)$ to $(18 a)-(18 d)$ is justified. The $S_{0} P$-isomer accumulation studies cannot distinguish between $\mathrm{S}_{1}-\mathrm{S}_{1}$ inter-isomer transfer (rates $k_{\mathrm{NP}}^{1}$ and $k_{\mathrm{PN}}^{1}$ ) and $\mathrm{S}_{1}-\mathrm{S}_{0}$ inter-isomer relaxation (rates $k_{\mathrm{NP}}^{10}$ and $\left.k_{\mathrm{PN}}^{10}\right)$.

For $\lambda_{L}=488$ and $514 \mathrm{~nm}$ the pump laser intensity was high enough (see fig. 3 ) to fit the P-isomer absorption cross sections $\sigma_{\mathrm{P}}$ at these wavelengths (circles in fig. 6a).

The functional dependences of $T_{\mathrm{pr}}$ on $\sigma_{\mathrm{P}}, \phi_{\mathrm{NP}}^{\mathrm{tr}}$, and $\phi_{\mathrm{PN}}^{\mathrm{tr}}$ are illustrated by the solid curves in figs. $4 \mathrm{a}-4 \mathrm{c}$, respectively.

The maximum mole fraction of $\mathrm{S}_{0}$-state $\mathrm{P}$ isomers, $x_{\mathrm{P}, \max }^{0}=N_{4, \max } / N_{0}$, which is reached for high pump laserintensities (eq. (21a)) is displayed versus excitation wavelength in fig. $6 \mathrm{~b} . x_{\mathrm{P}, \max }^{0}$ decreases in the region of dominant $\mathrm{P}$-isomer absorption (molecules in $\mathrm{S}_{0} \mathrm{P}$-isomer state are transferred to the $\mathrm{S}_{0} \mathrm{~N}$-isomer state via excitation to the $S_{1}$ state).

The ratio of the absorption coefficients $\left[\alpha_{\mathrm{P}}\left(\lambda_{\mathrm{L}}\right) / \alpha_{N}\left(\lambda_{\mathrm{L}}\right)\right]_{\max }$ in the case of $I_{\mathrm{L}} \rightarrow \infty$ is included in fig. 8. In the long-wavelength region the P-isomer absorption dominates. In this region DODCI in ethylene glycol is applied as saturable dye in picosecond and femtosecond rhodamine $6 \mathrm{G}$ dye lasers [1-9]. The bleaching of the P-isomer absorption plays the dominant role in the mode-locking process.

The absolute and relative accumulation saturation intensities versus excitation wavelength are plotted in fig. 9. Eqs. (22) and (23) are applied. In the long-wavelength region beyond $610 \mathrm{~nm}$ a steady-state P-isomer accumulation up to $x_{\mathrm{P}, \max }^{0}=0.5$ is not possible and therefore no $I_{\mathrm{S}, \mathrm{P}}^{0.3 b 3}$ values exist.

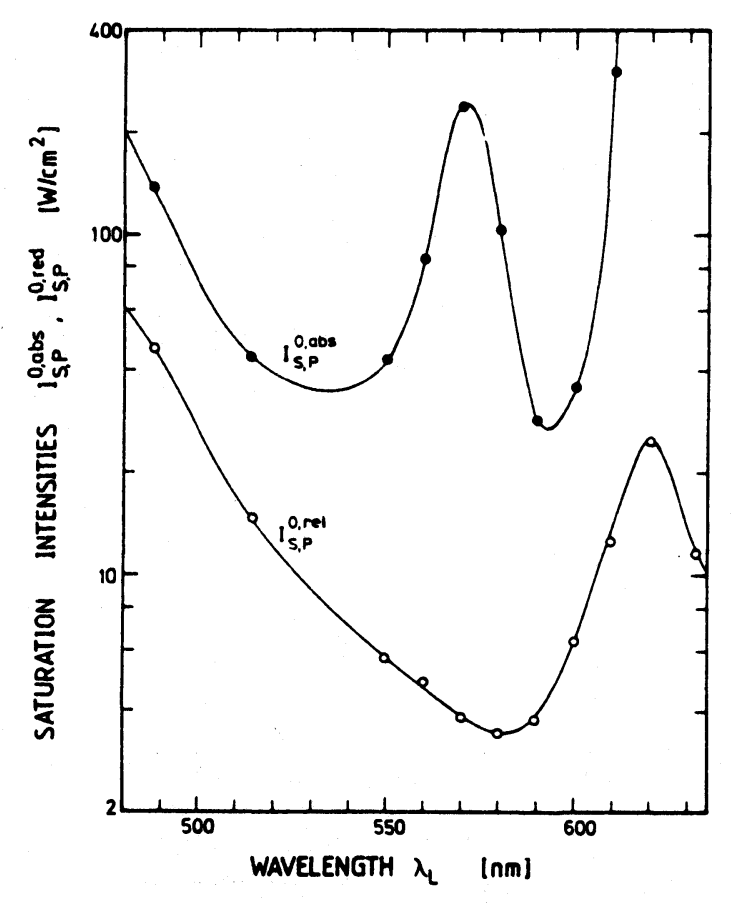

Fig. 9. Absolute, $I_{S . P}^{0.00}$, and relative, $I_{S . P P^{0}}^{0.9}, \mathrm{P}$-isomer accumulation saturation intensities of DODCI in ethylene glycol versus excitation wavelength. 


\section{Conclusions}

The laser induced accumulation of $P$ isomers in the $S_{0}$ ground state was studied for the dye DODCI in the solvent ethylene glycol. Computer simulation of the accumulation experiments allowed the determination of the photoinduced $\mathrm{N}$-isomer to $\mathrm{P}$-isomer and $\mathrm{P}$-isomer to $\mathrm{N}$-isomer transfer efficiencies. These transfer efficiencies are found to be strongly dependent on the excess energy of the excited molecules in the $S_{1}$ state.

\section{Acknowledgement}

The authors are indebted to Dr. F. Schneider of Spectra Physics for the loan of the argon ion laser. They thank the Deutsche Forschungsgemeinschaft for financial support and the Rechenzentrum of the University for the allocation of computer time.

\section{References}

[1] W. Schmidt and F.P. Schäfer. Phys. Letters A 26 (1968) 558.

[2] D.J. Bradley, in: Topics in Applied Physics, Vol. 18. Ultrafast Light Pulses, ed. L.S. Shapiro (Springer, Berlin, 1977) p. 17.

[3] E.P. Ippen. C.V. Shank and A. Dienes, Appl. Phys. Letters 21 (1972) 348.

[4] C.V. Shank, in: Topics in Applied Physics, Vol. 1, 2nd revised Ed., ed. F.P. Schäfer (Springer, Berlin, 1977) pp. 5.

[5] C.V. Shank, in: Topics in Applied Physics, Vol. 60. Ultrashort Laser Pulses and Applications, ed. W. Kaiser (Springer, Berlin, 1988) p. 5.

[6] R.L. Fork, B.I. Greene and C.V. Shank, Appl. Phys. Letters 38 (1981) 671.

[7] J.C. Diels, W. Dietel, J.J. Fontaine, W. Rudolph and R. Wilhelmi, J. Opt. Soc. Am. B 2 (1985) 680.

[8] J.A. Valdmanis and R.L. Fork. IEEE J. QE-22 (1986) 112.

[9] M.D. Dawson, T.F. Boggess, D.W. Gravey and A.L. Smirl. Opt. Commun. 60 (1986) 79.

[10] D.N. Dempster, T. Morrow, R. Rankin and G.F. Thompson, J. Chem. Soc. Faraday Trans. II (1972) 1479.

[11] C. Rulliere, Chem. Phys. Letters 43 (1976) 303.

[12] J. Jaraudias, J. Photochem. 13 (1980) 35.

[13] S. Rentsch, E. Döpel and V. Petrov, Appl. Phys. B 46 (1988) 357.

[14] S.P. Velsko, D.H. Waldeck and G.R. Fleming, J. Chem. Phys. 78 (1983) 249.

[15] O.E. Martinez, R.L. Fork and J.P. Gordon, J. Opt. Soc. Am. B 2 (1985) 753.

[16] V. Petrov, W. Rudolph and B. Wilhelmi, Rev. Phys. Appl. 22 (1987) 1639.

[17] W. Bäumler and A. Penzkofer. Chem. Phys. Letters 150 (1988) 315.

[18] W. Bäumler and A. Penzkofer, Chem. Phys. 140 (1990) 75.

[19] G. Angel, R. Gagel and A. Laubereau, Chem. Phys. 131 (1989) 129.

[20] A. Penzkofer and W. Blau. Opt. Quant. Electron. 15 (1983) 325.

[21] T.F. Johnsston Jr., R.H. Brady and W. Proffit, Appl. Opt. 13 (1982) 2307.

[22] J. Stoer, Heidelberger Taschenbücher, Vol. 105. Einführung in die Numerische Mathematik I (Springer, Berlin, 1972) p. 132. 